\title{
BURN INJURY
}

nature reviews disease primers
Burn injuries can be caused by
friction, cold, heat, radiation, friction, cold, heat, radiation,
emical or electric sources, but the majority of burn injuries are caused by heat from liquids, solids or fire.

\section{MANAGEMENT}

Five phases of wound care have been described. Phase I involves the immediate care for the patient, beginning with stopping the exposure, moving the patient into a safe area and flushing the injured site site with cool water. Initial assessments establish the state of the airway, circulation and cardiac function, disability, neurological deficit, gross deformity and degree of exposure. A definitive evaluation of the burn severity including size and depth

is also performed early in

the course of treatment as this influences subsequent treatment decisions. Phase ll focuses on fluid resuscitation to address hypovolaemia. In phase III, coverage of the wounds take place (for example, using skin grafts) to promote healing and reduce the

risk of infection; this typically occurs as soon as possible within hours to days after injury. Phase IV focuses on supportive or critical care, optimizing nutrition and medications to improve healing and reduce infection risk. Phase $V$ focuses on rehabilitation, which includes physical and mental health support to enable the person to return to regular life, address chronic pain and address psychological trauma.
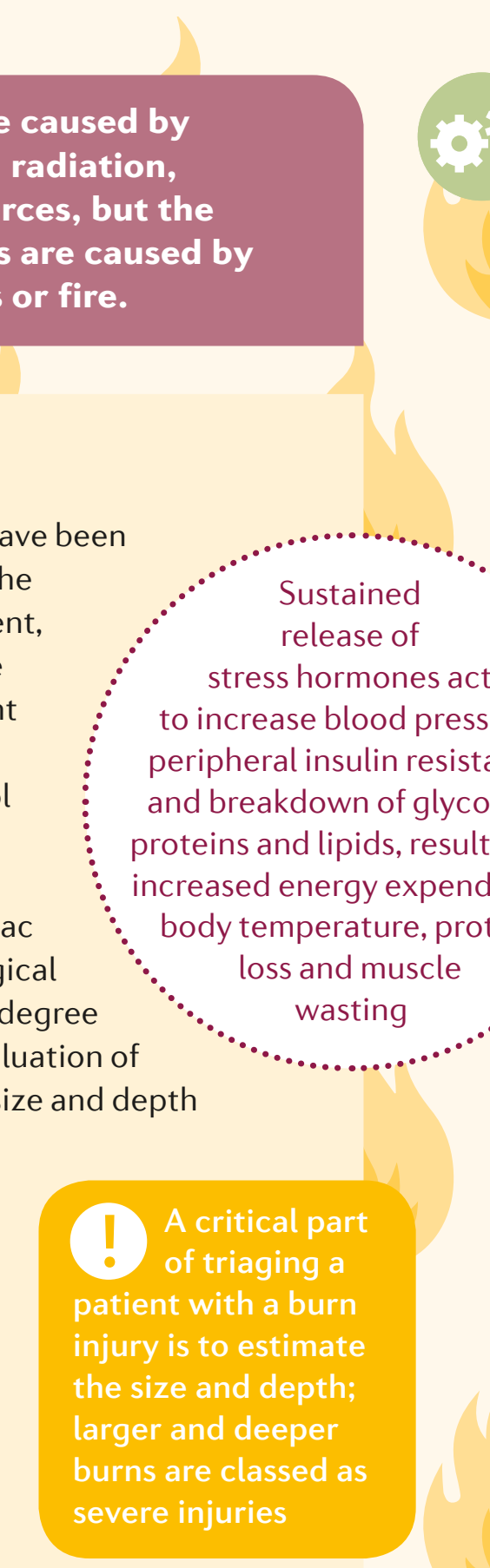

release of stress hormones act to increase blood pressure, peripheral insulin resistance and breakdown of glycogen, proteins and lipids, resulting in ncreased energy expenditure, body temperature, protein loss and muscle wasting

DIAGNOSIS

Superficial (first-degree) burns affect the upper-most layer of the skin, becoming red and painful. Superficial partial and deep-partial (second-degree) burns are less painful (owing to destruction of pain receptors),

Burn injuries trigger a complex set of responses, the sequence of which and how they interact is unclear. The more severe the burn, the greater the magnitude of the response.

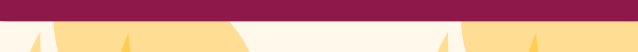
NITIAL INJURY Initially, an inflammatory response is triggered with the aim of healing the wound
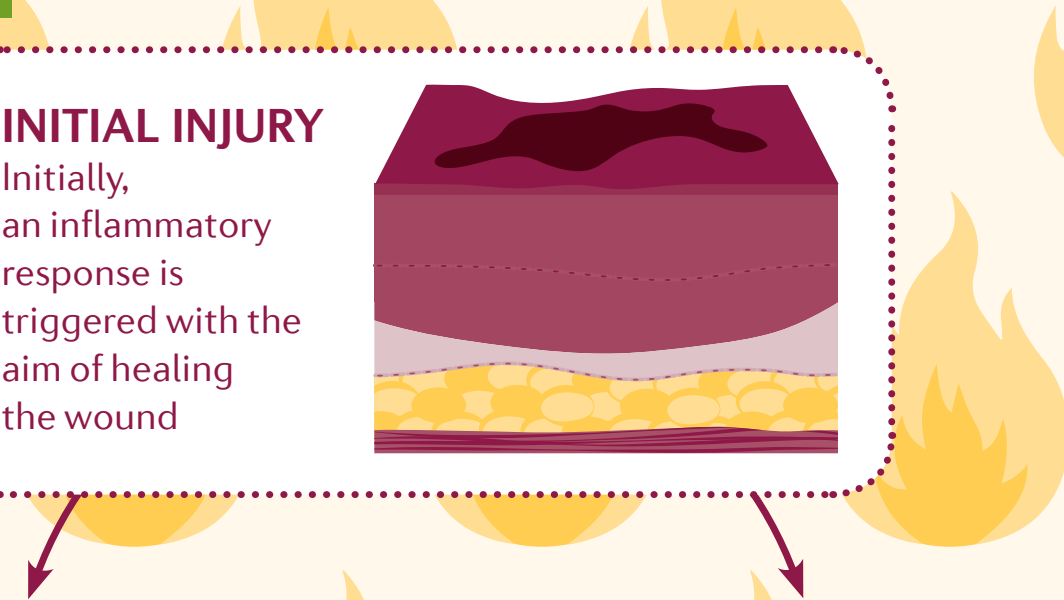

METABOLIC STATE $\sqrt{N}$
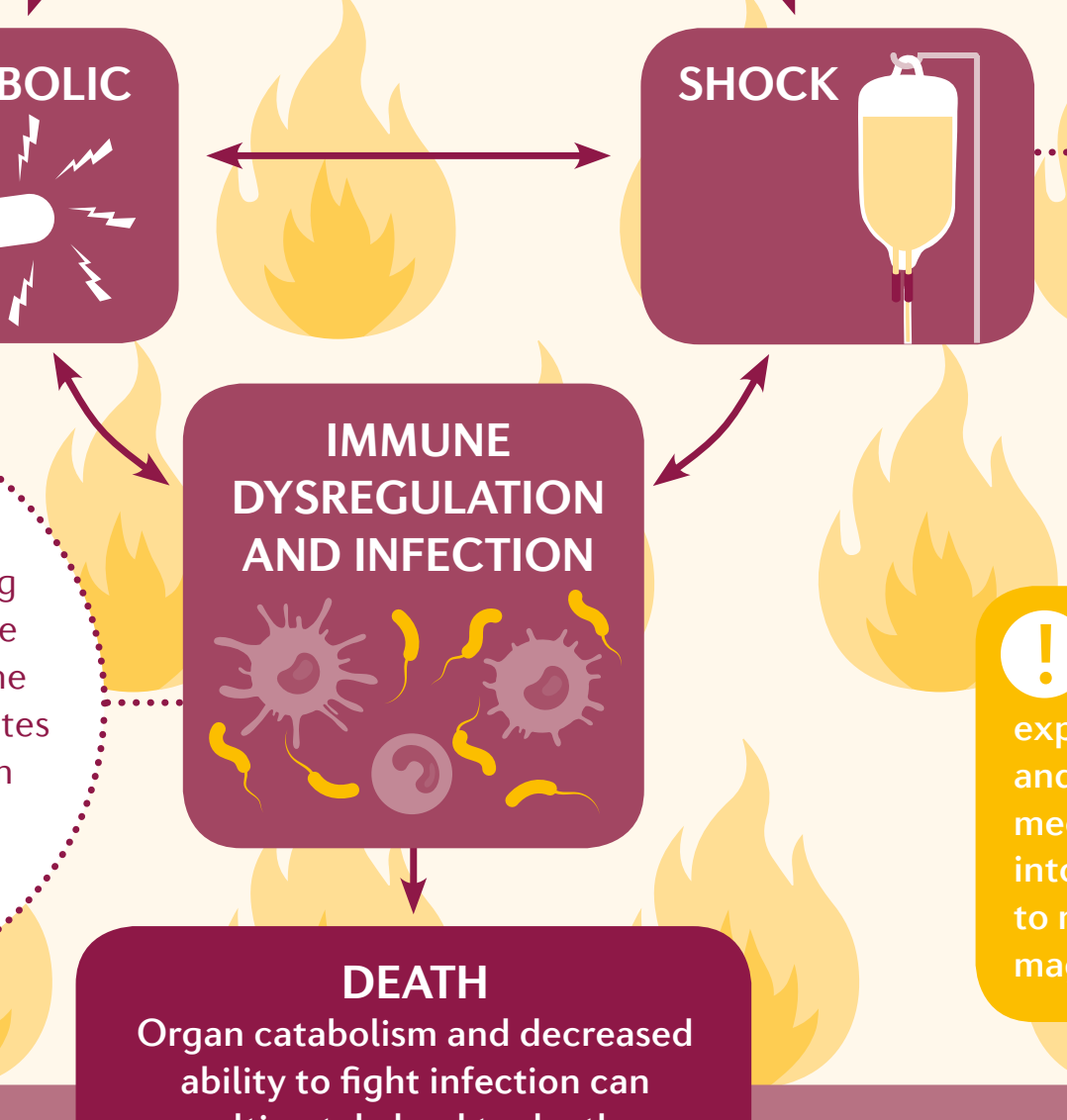

IMMUNE

DYSREGULATION AND INFECTION
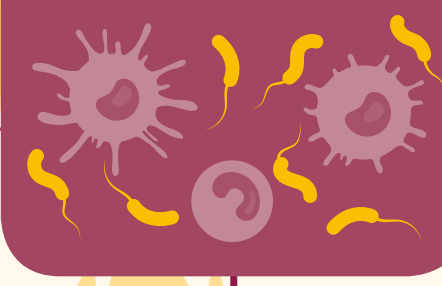

DEATH Organ catabolism and decreased bility to fight infection can ultimately lead to death more likely to require surgery and more likely to scar the deeper they are. Full-thickness (third-degree) burns extend through the dermis and are not typically painful owing to damage to the nerve endings.
For the Primer, visit do: 10.1038/s41572-020-0145-5 PREVENTION
Fourth-degree burns involve injury to deeper tissues, such as muscle or bone, are often blackened, not usually painful (owing to damage to the nerves) and frequently lead to loss of the burned part.
The majority of burn injuries are preventable. Grassroots changes of lifestyle factors (for example, introducing safe stoves), legislative regulation (for example, on clothing flammability) and prevention programmes

(for example, introduction of fire-safe cigarettes) have reduced the incidence of burn injuries in high-income countries. In lower income The settings, local social factors must be taken into consideration to optimize inflammatory mediators involved in wound healing have systemic effects, causing capillaries to leak fluid, contributing to tissue prevention efforts; for example, access to smoke detectors might be limited, so ways to limit open-fire cooking could be prioritized. To drive this process, the WHO has started a global registry to aid data collection in low-resource settings to optimize where efforts should be placed.
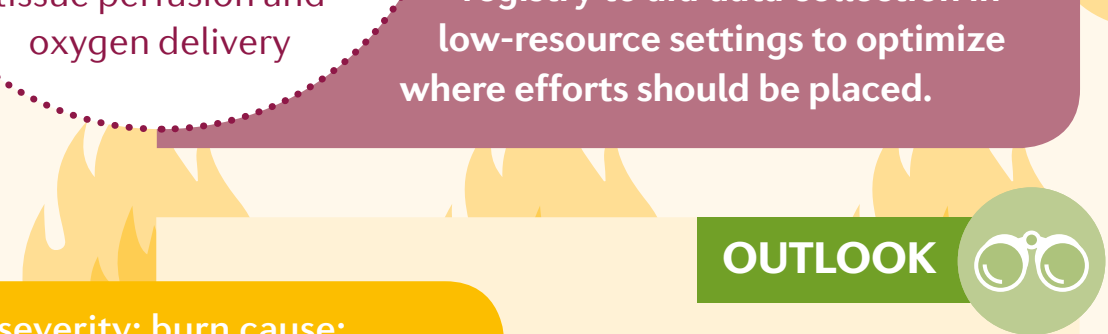

Burn severity; burn cause; inhalation injury; toxic exposures; other traumatic injuries; and patient age, pre-existing medical conditions, drug or alcohol intoxication and timing of presentation In the acute setting, artificial skin substitutes hold considerable promise for improved burn care, providing temporary or permanent coverage of open wounds. Skin substitutes should be resistant to shearing forces, have the structure and function of normal skin (for example, protecting from fluid loss and infection) and act as a scaffold to enable cells to proliferate for healing. In the long-term, burn survivors have a substantially greater mortality related to mental illness and decreased quality of life. Priority should be given to diagnose, treat and prevent these long-term morbidities and, therefore, long-term studies to gain better insights are urgently needed. 